\title{
Artificial Neural Network based Applications in Mechanical Engineering
}

\author{
Mohit Gangwar, Brijesh Kumar Ojha, Rohit Srivastava, Himanshu Kumar Shukla, Aditya \\ Pratap Singh
}

\begin{abstract}
Search of sheet metal components along with physical items calls for a good deal of expertise in addition to creating expertise on the part of designers. Lately, different Artificial Intelligence (AI) methods now are being used in sheet metallic labor to minimize complexity; take lower the dependency on male, time and also expertise ingested physical appearance of pieces and expires and additionally to enhance one success. Artificial Neural Network (ANN) method is by using the most effective materials for fixing engineering layout issues and minimizing errors to drop with experimental information within actual physical engineering. This specific investigate documents particulars a substantial comment of applications of ANN strategy to sheet metallic perform how about hand-operated engineering apps. Major printed analysis do inside the domain name of bodily engineering is really summarized. In line with the vital comment of accessible literature, a lot more analysis range is really determined. The present literature analysis uncovers that there's stern need in an attempt that you are able to make use of ANN means to press items style as well as in addition to foresee gear lifetime contained sheet metal industries or perhaps maybe even in for bodily engineering process.
\end{abstract}

Keywords : Artificial Neural Network, Mechanical Engineering, Artificial Intelligence, Sheet Metal, Machine Learning.

\section{INTRODUCTION}

Artificial Neural Network (ANN) is actually an incredibly rapid development of cross problem; it pertains to biology, mathematics, electronics, physics, psychology, computer science, cognitive science and many other subjects. It is the study of the adaptive programming info process of an

Manuscript received on April 02, 2020.

Revised Manuscript received on April 15, 2020.

Manuscript published on May 30, 2020.

* Correspondence Author

Mohit Gangwar, Dean-Engineering, Bhabha University, Bhopal, MP, India. Email: mohitgangwar@gmail.com

Brijesh Kumar Ojha, Asst. Prof., Department of ME, Faculty of Engineering, University of Lucknow, Lucknow, UP, India.

Rohit Srivastava*, Asst. Prof., Department of CSE, Faculty of Engineering, University of Lucknow, Lucknow, UP, India.

Himanshu Kumar Shukla, Assistant Professor, Department of CSE, KMC Urdu-Arbi Farshi University, Lucknow, UP, India.

Aditya Pratap Singh, Asst. Prof., Department of CSE, School of Management Science (AKTU Code 421), Lucknow, UP, India.

(C) The Authors. Published by Blue Eyes Intelligence Engineering and Sciences Publication (BEIESP). This is an open access article under the CC BY-NC-ND license (http://creativecommons.org/licenses/by-nc-nd/4.0/) engineering self-discipline, is actually a novice driver info processing technology [1]. The concept of it is on the basis of contemporary neuroscience analysis advantages, utilizing sizable amount of simulation strategy, sector storage, and parallel, attempting to reflect some of the basic characteristics of the human mind info processing. Along with the improvement of the basic principle and ideal, will of the improvement of smart manual engineering plays a much more essential role. Along with the usage of contemporary technology in addition to science on the device, the framework of contemporary manual equipment much more as well as more complicated, run much more progressively more is equally high level of automation. ANN has sizable program of the region of mechanical engineering. By the basis of this hardware engineering disciplines to the unit layout, manufacture \& operation, ANN engineering is actually pervasive [2].

Sheet metallic areas are vital structural individuals in automobile bodies, telecommunication equipment, home appliances, beverage cans, aircraft, and health implants. Sheet metallic methods are popular internal industries to produce the parts as the recurring electric capability of it's in addition to best effectiveness. Existing business demands that sheet metal pieces must be made to correct condition or perhaps near correct condition with enhanced actual physical attributes, high-quality dimensional accuracy, sleek surface finish as well as material price savings based on service issues. Sheet metallic developer has to see a choice of problems as well as proper appearance of press equipment to create places without any surface area or maybe perhaps internal defects, lower manufacturing cost, least scrap, higher efficiency in addition to electricity use [3].

Sheet metallic portion is actually common in automobile industry as well as aviation business because of the improved strength of it is and light weight. Nevertheless, sheet metal part typically is going to deform for machining, assembly, as well as calculating stages all through the whole production process because of the characteristics of its of slim framework, size which is actually big, together with little rigidity to constrain remarkable sheet metallic component deformation [4]. Regular approaches of look of sheet metal parts as well as media equipment are highly, laborious, and complex impacted by male knowledge. 
Only a bit of Computer Aided Design (CAD) in addition to Computer Aided Engineering (CAE) methods is really used in sheet metal industries since previous couple of years to relieve intricacy minimizing moment. Even though the choices offer assistance for page layout, drafting, creating along with analysis of sheet metallic strategies, but domain title gurus constantly be made to shoot choices during almost all phases of layout and manufacturing [5].

Throughout previous 10 to 15 yrs, different Artificial Intelligence (AI) methods are in fact used for fixing issues which are complex to come down with just about all components of engineering. Lately, a good deal of researchers similarly have used ANN method to AI for manufacturability analysis, run out page layout, process planning, prediction of limited component simulation strengths etc. contained sheet metallic provide the outcomes [6].

Sheet metal merchandise development \& color of expires usually calls for many like information. A little alter of each parameter comprises a new including situation and groundbreaking simulations have to value the common overall functionality of it is and also actions. It's not helpful to search for the correct choice by means of one-by-one simulation. To be in a position to handle this certain problem, ANN approach coupled with Finite Element Method (FEM) may be utilized efficiently for sheet metal merchandise development as well as look of expires to discover the truly non linear link including the layouts details [7].

ANN tactic sticks out there since the duplicates of male mind. ANN methods are in fact fusion of several basic processing pieces which are used in parallel. ANN technique is able to elevated functions, like learning or adaptation, input result mapping, and fault tolerance, non liner function together with decreased health level works for example info pre processing for a selection of types of inputs. ANNs are in fact affected both by male natural nervous means as well as mathematical theories of learning, information processing along with sense of balance one. ANN performs issue on the various kinds of purposes. For example, X1, X2, X3----Xi because the inputs with regard to $\mathrm{W} 1$, W3----Wi since the weights of the feedback functions. All of the inputs into information are in fact represented by linear mixture of the outcome [8].

Anywhere, for example, $\mathrm{j}$ is going to be the correspondence site link in between neuron as well as computation neuron node is in fact i. ANNs are in fact nonlinear functionality processing buildings in which the components referred to as the information is actually point to do by neurons. Indicators are in fact transmitted of key in to papers that is really recognized as connecting returned once again back links. The hooking links are in fact connected to extra fat that is multiplied with the completely new signal i.e. overall reviews for almost any regular neural telephone system. The paper signal is really acquired by using activation to the web feedback. An ANN is really put in place for a specific program, for example pattern recognition or maybe information distinction, by technique of a mastering efficiency. Learning within answers which are actually all natural options switches in the synaptic junctions accessible regarding the neurons.

In this particular analysis paper, accessible published studies function in the region of applications of ANN technique to sheet metal perform and in the domain name of mechanical engineering is actually evaluated. Salient qualities of several essential techniques produced by worldwide scientists are actually presented; the range of extra task of sheet metal is actually determined [9]-[11].

\section{BACKGROUND}

Typically sheet metal work is actually laborious, period intensive, complex as well as skilled based procedure. Uses of ANN strategy to sheet metal jobs have furnished a groundbreaking dimension in the late 1990 \& fast 1995 . ANN strategy to sheet metallic entails process planning and it is to be used up web page layout, prediction of FEM benefits etc. There're crucial factors of sheet metal merchandise advancement. Approach preparation establishes the values of a selection of system details, information of capabilities, strip system design \& style etc. The program of activity of kick the container webpage page layout, customized seems to have to figure out the kind as well as scale of a selection of kick the bucket areas, the modeling of theirs as well as elements decision. Globally scientists pre owned ANN mode of development of unique remedies to ease the tasks regarding sheet metallic provide the outcomes [12].

Researchers [13] recommended ANN used qualified process for sheet metallic bending tooling. Based upon understanding and examining comprehension of networks, the info acquisition additionally to comprehending inference to the guru process is really used. They divided learning sort straight into two groups based on the problem of theirs qualities; conditional qualities together with electric qualities. A conditional attribute is really used similar to a mastering variation.

Author [14] demonstrated sheet metallic bending with ANN. ANN is really utilized to foresee the forces required for a selection of bending assessments and furthermore to attain a spring back viewpoint. Researcher [14] pre owned Multi level Perceptron (MLP) neural networks with rear propagation mastering algorithm. Hyperbolic tangent is really utilized as activation efficiency.

Authors [15] unveiled fuzzy set concept, ANN combined with particular telephone system on the format info of this particular stress as well as strain a component of progressive kick the container structure. For color of progressive kick the pail, different variables are in fact believed remaining as kick the container obstruct thickness, kick the container clearance viewpoint, stripping stress, kick the bucket in addition to run out with hand operated bushing etc. Just as before propagation algorithm is really useful for instruction.

Authors [16] Experts proposed FEM along with ANN to reduce springtime refunded within a channel being produced procedure. Via FEM view they regarded a few of problems like supplies attributes, processing information, tooling geometry along with lube circumstances as well as sheet thickness. Multi-layer feast forward ANN with Back Propagation Algorithm (BPA) is actually used. 
Authors [17] created ANN for cost evaluation of huge level construction of sheet metal parts. For the type of yours key in amount of ANN they regarded as volume of strokes, volume of gear phases, as well as amount of border parts and stamping. For the mastering unbiased easily propagation algorithm is used.

Authors [18] proposed mixture of ANN combined with the elementary plasticity idea for identification of elements traits and lube express to the total style process. They regarded as enter into information for example punch great deal, punch stroke, blank positioning strain, flange thickness anxiety, flange minimization ratio etc., consequently the information of ANN as unique plastic substance an isotropic coefficient, plastic-made made coefficient, along with labor hardening exponent etc. Once more BPA is used.

Authors [19] use ANN to air bending procedure. They deemed key in color of ANN as twist perspective right after unloading, sheet thickness, real bodily measures of this information, bending punch and emotional stress, displacement together with the newspapers color of ANN is really punch stroke. They used returned once again bipolar sigmoid propagation algorithm activation efficiency.

Authors [20] gave mixture of ANN in addition to wireless inkjet printer mastering techniques for region defect searching found sheet metallic acquiring. They deemed sex variables for region defect as degree gradient, boundary of torsion gradient, torsion went, boundary of curvature gradient, boundary of degree gradient, curvature gradient etc. Logistic linear threshold as well as hard restricting (on/off) are actually as activation effectiveness and also nourish forward once more in BPA ANN is actually used.

Authors [21] proposed ANN kind for prediction of blanking development in addition to blanking particulars by looking at Acoustic Emission (AE) as well as power waves. Pros utilized AE receptors, Digital Signal Processing (DSP) hardware along with acquisition software program for amount AE signs along with power signs. Pros seen as volume of ANN enters particulars including kick the bucket. The punch advantage of profile together with the punch kick of the pail by $\mathrm{AE}$ waves along with power signs. Once more BPA is used. Pros used specific system known as NeuroShell due to the ANN designs as well as to have the ability to evaluate them. with many of this command of springback phenomena in the bending practice of light weight aluminum sheets by crossbreed developing procedure. Metal substrates are actually pre bent to nominal types during a built-ad-hoc module immediately after getting constrained on it. Next, Authors [22] had been post resolved by improved power diode laser to give up the deformation of pre bent sheets sticking with the release of the limits. The assortment of springback phenomena are actually approximated by computing the massive difference involving the nominal bending perspectives in addition to individuals carried out on the unconstrained substrates next laser beam blog post remedies. Analytical designs, certain for forecasting the springback by altering the surroundings of the purposeful details of the developing procedure, are created. Neural society methods had been in addition advised to boost the
Authors [22] presented the study of theirs which functions

matching among numerical and experimental information, with the MLP taught by BPA being probably the fittest one. Because of this foundation, a management modulus very helpful to providers for automation as well as simulation purposes was developed on. The exploration pertains to the experimental assessment as well as analytical modeling of laser beam assisted bending procedure of AA6082 T6 very little aluminum slim sheets. The pattern of aluminum used largely on starting deflection as well as laser beam purposeful parameters enables to discover the best physical exercise window with the laser beam assisted bending procedure to compensate the springback with no taking on within therapy and that is in fact inadequate or perhaps maybe perhaps undesired winter time of year alterations of the prepared substrates; based on the option of this particular laser beam operational info, five different scenarios is really identified:

(i) Ineffective treatment for severely little selection along with fluency of passes.

(ii) Partial compensation of this particular springback for moderate or poor quantity along with fluency of passes.

(iii) Proper compensation of this particular springback for intermediate fluency additionally to quantity of passes.

(iv) Over bending for a lot of quantity in addition to fluency of passes.

(v) Massive winter season alterations of this substrate for gravely taller amount along with fluency of passes.

The phenomena associated with the kind adjustment by laser beam articles treatment of the substrate subsequent to physical bending when nonetheless constrained on the kick the container are generally ascribable to two special reasons:

(i) Inhibition of springback by discerning heating as well as concurrent annealing of perhaps the topmost ph amounts on the substrates continue to below constrain.

(ii) Extra bending of constrained metal substrate caused by wintertime gradients of the canon power shot in the pre bent article all through the laser articles therapy.

\section{RELATED WORK}

Neural and empirical society version may be profitably used to control as well as limit the springback within laser beam assisted producing technique of $\mathrm{V}$ shaped small aluminum slim sheet, with MLP neural networks presently being maybe the fittest airier; MLP neural networks are actually discovered to have outstanding generalization ability similarly as part of interpolation and within extrapolation, when they're employed to foresee light industry aluminum outside the quantity they would been taught for. To sum up, neural society cures can be quite obtainable in predicting, managing as well as managing springback of $\mathrm{V}$ shaping of finer light weight aluminum sheets. AI is consequently an useful choice to finite and analytical component models. It is in a place to enable the significance of sensible power for automation as well as therapy monitoring as well as facilitate the enhancement of original approximation modulus very helpful to the practitioners as a result of the command of bending procedure of employment piece with essential geometry [23]. 
In this specific exploration newsprint a male made ANN is actually used to foresee the thickness round a cup wall structure system process of hydro physical abundant drawing.

This specific style is determined by a feed forward refunded prorogation neural phone feature. Immediately after employing the experimental outcomes to instruct as well as assess was positioned on to absolutely brand new details as a result of the prediction of thickness strains in only hydro physical abundant drawing. The results are actually promising. Within the existing task, we in addition attempt to complete a restricted component simulation of the process for the 2 dimensional symmetric situation making use of explicit restricted part code LS DYNA 2D. Counter strain on the blank is actually used by indicating the stress boundary difficulties. A comparison was produced between simulated, experimental together with ANN results of hydro physical severe drawing utilizing genuinely little carbon extra severe like quality metal sheets of $0.96 \mathrm{~mm}$ thickness. It was additionally found that by hydro physical abundant drawing, greater draw ability in addition to a much more in reality thickness division had been received in comparison with conventional primary drawing. It has been discovered the hydraulic counter strain severe drawing procedure has benefits that are a selection of conventional abundant drawings. In this process draw ability of sheet metal is often produced better as a direct result of higher Limiting Draw Ratio (LDR) that may be obtained as in comparison to conventional primary drawing. Experiments done on extra severe Drawing Steel (EDD) sheets proved LDR construction projects the consequences greatly. The process was simulated keeping utilization of explicit limited component code LS DYNA 2D. The simulated outcomes had been in excellent understanding with experimental ones. LDR fused with thickness strains are actually anticipated with sensible precision. Thus hydro physical abundant like might be additionally simulated diet LS DYNA 2D. Quality things which are great could be reached by counter worry. Thickness division of EDD cups for neural world advantages, experimental and simulated outcomes are in fact for counter strain really impressive look at LDR excellent drawing because of to a good deal a lot more constant division of thickness nervousness as in comparison to conventional primary drawing. The experimental verification accomplished due to the information examined for the prediction of yours of this specific beginning of localized necking within the conventional and also hydro physical serious drawing methods demonstrates extraordinary agreement with simulation along with experimental results. A neural network layout was created in addition to educated by experimental specifics sets. The inputs of the device were material and moreover, the output \& system parameters were absolutely thickness strains with the cup wall structure system. The competent neural community was positioned on to info that is brand new to foresee the thickness division. The neural network works quite well which allows it to foresee a huge component of strains properly. And so the application of ANN can increase the knowhow of this particular hydro physical serious drawing procedure [24].

The wipe bending is just one of things by far the most frequently put on to the sheet metallic merchandise enterprise.

Furthermore, the springback of sheet metallic that's referred to as adaptable restoration of the issue all through unloading should be checked out to produce bent sheet metal ingredients within proper tolerance limits. Springback is influenced by the elements like sheet thickness, lubrication circumstances, tooling geometry as well as material traits as well as processing parameters. In this specific exploration paper, the prediction variety of springback using wipe bending treatment was developed by using ANN technique. Below, several numerical simulations utilizing FEM had been carried through to get the teaching info of neural networking. The learned neural society is numerically analyzed and might be immediately used springback prediction for new instances. In this specific research paper, based on info out of Finite Element Analysis (FEA), the prediction variety of springback was developed by neural phone system. The results teach the consistency between the networking as well as simulator version benefits is actually accomplished by relative error below $0.8 \%$ as well as nine \%, respectively. It is considered that a properly trained neural society design provides fast and results which are constant, making it an easy program in preliminary studies for that type of engineering complications. This particular method at the research paper might be taken into account as an alternative, quickly and pragmatic method to evaluate the car parameters [25].

In sheet metallic industries, the ability to foresee and also stay away from issues, such as necking, fracture as well as wrinkling are actually of great worth. It is important to work within the protected strain region to avoid these failures. The Forming Limit Diagram (FLD) is actually thought the best great program to get the protected strain location for every sheet metal in a variety of strain circumstances as well as ration. In this specific research newspaper [26], significance of vital process parameters especially, punch velocity, Blank Holder Pressure (BHP) and temperature on FLD associated with a Ti-6Al-4V alloy are actually examined. Taguchi technique was used to figure out the effect of the parameters on little strain and substantial stress. The limited component variety of extreme drawing procedure is made up and examined using Dyna form version 5.6.1 with LS Dyna design 971 as solver. The simulations are completed in Taguchi order to receive the values of tiny strain and big stress. Furthermore, the values of major and minor strain is actually predicted utilizing ANN worrying regarding type in details as punch velocity, BHP and also heat as well as normalized distance of every cup. Growing limit curve is actually drawn by making use of Keeler's equation thinking regarding the characteristics of Ti-6Al-4V at various forming temperatures. It's discovered the expected strain values are going to be in excellent agreement with the experimental information. The ANN style has an excellent capability to foresee this info along with the very same might be observed along with the correlation coefficient well worth (R) which is near to 0.99 for every major and minor stress. The comprehensive typical error for prediction of major and minor strain is actually $4.2 \%$ coupled with $1.7 \%$ respectively.

Published By:

Blue Eyes Intelligence Engineering \& Sciences Publication 
As a result, ANN might be properly used to keep away from experimental consider outs in addition to simulations to have discover of this formability of Ti-6Al-4V sheet.

This specific research newspaper [27] details an approach used on ANN to figure out the components parameters associated with a stainless material. The experimental method of the bulge test is used to determine the components reply below loading. The ensuing strain displacement curve is actually transferred to certain neural network which had been educated utilizing strain displacement curves created by restricted part simulations of the bulge examine and the corresponding materials parameters. Through an instruction activity the neural system produces an approximated function for the inverse concern relating the parts information on the type of the strain displacement curve of this bulge evaluation. The bulge talk with a circular die is actually used to figure out the stress hardening curve, the individual having an elliptical kick the bucket for an off of axis viewpoint is actually utilized to determine the Lankford's coefficients in addition to the one particular having an elliptical die for an off axis perspective of is actually used because of the validation of this content material parameters' identification. The latest program is produced to figure out the material characteristics of stainless steel articles by exploiting the experimental bulge assessment results. ANN is able to earn elliptical kick the bucket for an off axis viewpoint of forty five. Approximated function for the content material parameters based on the type of the strain displacement curve of the primary point of the clear. This specific characteristic is actually acquired during an application activity with outcomes from numerical simulations. Then, it's exploited due to the identification of components parameters coming from experimental results. ANN can readily predict material details with dependability that's suitable for a lot of style things to consider. It should be mentioned that after ANN was knowledgeable, output results for a particular range of enter info may be discovered very quickly.

Fixture plays an immensely crucial component in constraining a lot of sheet metal component deformation at giving machining, assembly, as well as calculating stages all through the whole production process. Nevertheless, it is nevertheless a difficult and nontrivial process to build as well as improve sheet metal fixture locating layout at current since there is typically less explicit as well as immediate phrase describing sheet metallic fixture finding structure and responding deformation. To that conclusion, a Radial Basis Function (RBF) neural society prediction layout is actually recommended to this particular research paper to help you and also look of sheet metal fixture finding layout. The RBF neural program design is built by instruction info set selected by actually sampling and limited component simulation evaluation. Finally, a case study is actually carried out to verify the suggested method. In order to assist the layout along with sheet metal fixture finding format, this specific newsprint establishes an RBF neural society prediction layout for describing the mapping link between sheet metal fixture finding platforms and responding deformation. The primary contributions of the analysis paper are actually the following [28]:

(i) A prediction layout determined by RBF neural society for sheet metal fixture finding layout design coupled with produced, and the prediction accuracy fulfils the need of widespread engineering.

(ii) As soon since the proposed prediction style is actually utilized to fixture locating layout a couple of yrs down the line, it's in a position to modify the minimal part simulation for sheet metallic deformation. Thus, the calculation measurement is reduced and consequently the usefulness of fixture locating layout design coupled with improved.

(iii) In difference to the rear propagation neural society knowledgeable as well as tried out with precisely the exact same evaluation sets, the RBF neural society based prediction style is actually of higher accuracy in addition to much more sound.

Prediction of living of combination die is actually an important exercise typically performed by expert die designers in deep sheet metal industries. In this specific research paper, searching labor energetic within the prediction of livelihood of compound die by using ANN is actually provided. The details impacting lifetime of combination die are actually examined by FEM evaluation and additionally the essential simulation values are actually pushed. And then, an ANN style is created by utilizing MATLAB. This ANN design is competent from FEM simulation advantages. The proposed ANN style is analyzed efficiently on different compound dies made for producing sheet metal pieces. A test run of suggested ANN style is actually displayed to this specific paper. In this particular analysis paper, an ANN layout was developed for prediction of lifestyle of compound die. The method used for prediction of die living will continues to be talked about briefly. The suggested ANN design [29] works well at accomplishing the tedious and time-consuming task of prediction of lifestyle of compound die in an incredibly short time period. The device was examined effectively on various compound dies taken of sheet metal industries. A sample run on a typical compound die demonstrated the comfort of the suggested design. The same variants might also be produced for prediction of living of some other sorts of dies like progressive die, abundant drawing die as well as bending die.

In this specific research paper, experts [30] article on the advanced and used of restricted component methods, are actually created as well as used for sheet being created simulations ever since the 1970s, and also have immensely contributed to make sure the achievements of concurrent appearance of the generation actions of sheets metallic of the developing functioning, the Gurson-Tvergaard-Needleman (GTN) layout was typically utilized to evaluate the ductile damage in addition to fracture phenomena. GTN represents among probably the most famous ductile damage model. In this specific research paper, many experimental examinations \& minimal component model computation are actually carried out to foresee the damage evolution in just notched tensile specimen of sheet metallic using the GTN layout. 
The variables within the GTN layout are actually calibrated using an ANN system and furthermore, the results of the tensile evaluation. In the experimental portion, authors [30] used optical measurement equipment in 2 phases: firstly all over the tensile evaluation, a digital photo correlation strategy is placed on to determinate the complete area displacements of the sample top.

Then a profile projector is actually utilized to evaluate the localization of deformation correctly ahead of when the specimen's fracture. In the validation ingredients of in this particular analysis paper, the experimental outcomes of hydro being created part in addition to Erichsen test are actually in comparison to their numerical restricted component layout thinking about the GTN layout. A really good correlation was discovered in between the 2 strategies. In this particular analysis paper, the GTN layout was utilized to evaluate the ductile damage in addition to fracture phenomena. An identification process based on ANN is actually used to determine the parts parameters of GTN harm model. A back propagation training neural society layout was told by using the restricted component advantages of notched tensile test with several of the destruction details. A much better choice of the concordance as well as ANN framework on the inputs allows producing better ANN layout. The optical metro logy as well as exclusively the method are rapidly becoming very popular in illumination of insightful the accessibility as well as free results of company software package and hardware. Through these methods of measurement we find the displacements in many points on specimen surface as well as we look at thoroughly the experimental results of shear band growth along with the thickness deviation within bulged part with the numerical results. The Identified details applied to the Finite part kind of acquiring operation, these outcomes had been in excellent understanding with experimental ones. To follow this comparison, experts [30] can summarize that the technique of ours is actually going to be completely ready to make a reliable outcomes and refreshing efficacy for the identification of damage parameters. The suggested program based on minimal component which will come with ANN is usually used to have the ability to add towards the sheet metallic characterization, however with a lessened computing phase in comparison to the classical inverse identification plans. On the flip aspect extension of this specific method to the design and style accompanied by an isotropic yield criterion is actually going to be also investigated.

The sheet metal bending is actually an important sort of sheet metal forming process, widely used in various manufacturing applications as aircraft, energy industries, cars as well as home items. This specific exploration analysis predicts the reactions of this sheet metal bending technique by using ANN. Based on cases look at reducing limited component method, a neural method was trained. Sheet thickness and expire radius were definitely the feedback; stresses, strains, springback, and loads, etcetera were definitely the outcome for the neural program. The competent neural community was evaluated for 5 new patterns. It was learned that the vast majority of the outcome was somewhat close to the simulation advantages. Such a technique of reaction prediction aids in reducing the, computational period. In this specific research paper, responses of sheet metal bending methods are actually expected making use of artificial neural phone system. The neural method is trained, determined by the results of forty 4 cases evaluated using the restricted component methods. Sheet thickness and expire radius had been viewed as enter and effective stress, residual strain, plastic-made stress, springback, residual anxiety. The competent neural community was evaluated for 5 new cases and in comparison to the limited component advantages. It was recognized the neural method offers quite near predictions of sheet developing reactions. This particular type of predictions will help to reduce big computational period entering computer simulations. It might be managed while by beginner in FCA [31].

In sheet metal being created springback is actually a trend which occurs rather due to recurring stresses of the content material, while bending the sheet metallic. Hence it ought to avoid enhancing the metal quality near the prediction of springback perspective. By forecasting the springback guidance, may bring down the point of view by altering the parameters. As a result, an excellent prediction technique is actually essential to foresee the springback perspective. Among the finest prediction techniques is actually the ANN to foresee the springback perspective of sheet metal. And thus in this particular research paper seeks to increase the prediction efficacy of ANN by combining particle swarm optimization (PSO) algorithm. The PSO algorithm is used to instruct the ANN; consequently it's in a position to anticipate the springback angle properly. The proposed tactic would be in contrast to the experimental outcomes along with the conventional prediction strategies such as Genetic Algorithm (Conventional ANN and GA) based mostly ANN. This research paper suggested a very efficient ANN technique to foresee the springback viewpoint of fresh bending with sheet metal developing process. The suggested approach exploited 2 ANN architectures, one particular with K-Value and n-value as inputs along with the many others with $\mathrm{R}$, $\mathrm{t}$ and $\mathrm{R} / \mathrm{t}$ as inputs. The intent of utilizing GA is actually overcoming the problems in regular BPA in such a fashion that the error was reduced to the small as compared to the regular methods. The validations outcomes have established the suggested PSO. ANN outperformed the standard procedures. This specific, consequently, directs the recommended ANN tactic is much more suitable for forecasting the springback viewpoint of wipe bending process [32].

The developing conduct of Tailor Welded Blank (TWB) is actually impacted by thickness ratio, energy ratio, as well as welds issues in a synergistic fashion. In the vast majority of the instances, these variables deteriorate the developing conduct of TWB. It is crucial to anticipate suited TWB trouble for accomplishing much better stamped merchandise made out of welded blanks. This is quite difficult also as useful resource intensive, needing great deal of tests or maybe simulations being completed under diverse base material also as weld issues. Auto sheet component designers are actually likely to be substantially gained if a specialized system' can be bought which could send creating behavior of TWB for combined weld and also blank factors.

Published By:

Blue Eyes Intelligence Engineering \& Sciences Publication 
This specific paper primary aims at creating an ANN layout to foresee the tensile conduct of welded blanks manufactured from steel quality along with lightweight aluminum alloy base materials. The crucial tensile attributes of TWB are actually expected within picked choice of weld situation and combined clear.

Around the project, PAM STAMP 2G restricted parts code is actually used to be able to imitate the tensile conduct and additionally to create output info needed for training the ANN. Predicted achievements from ANN design are actually compared also as validated with limited component simulation for 2 unique intermediate TWB issues. It's found the results from ANN are actually encouraging with proper prediction errors. A pro technique framework is recommended making use of the competent ANN for creating TWB situations that will deliver better formed TWB strategies. The following will be the outcome from the existing labor [33]:

(i) The typical tensile conduct of welded blanks would be in line with the current knowledge and understanding. For example, the modes of disappointment with appreciate to weld zone attributes, effect of weld orientation on the great deal requires along with elongation, influence of thickness ratio on the tensile activity coincides with the being sold results.

(ii) In total, the TWB tensile result parameters from ANN styles are actually in excellent agreement with the simulation results for selected base materials along with TWB issues. ANN can foresee the stress strain reply of welded blanks within acceptable error limits.

(iii) A pro procedure layout goes on to be highly recommended to foresee the developing conduct of welded blanks. This specific method which is actually utilized for extra simulations are required by evolution to account for many more material attributes, TWB circumstances, components styles together with some other forming behavior.

Punching is among probably the most essential sheet metal production tasks in mass production of metal regions along with elements of a great range of industries. Lately, an extra comprehension of technological facets of this punching process goes on to be acquired, providing the opportunity to incorporate the completely new insights in the ca effect of the manufacturing functioning.

This particular paper [34] indicates the completely new insights in the pounding progression as well as reveals how these findings might be employed in a process management concept. The utilization of an artificial neural society plays an important role in this specific concept. This specific research paper has offered brand new insights in technological aspects of the punching functioning. It has shown that an analysis of the Force Displacement graph may be associated with product quality. Important indicators are actually the job achieved and punch penetration inside the goal of optimum force. An on line measurement of the values supports the possibility for an automated and premature detection of quality problems. This specific research paper implies that ANN might be advantageous to value an automated monitoring in addition to diagnoses procedure. A concept for an autonomous procedure management process for pounding operations is usually recommended to this specific research paper. This might be considered a very helpful activity towards the integration of pounding functions in pc incorporated manufacturing environments.

Growing Limit Diagram (FLD) supplies the restricting strains a sheet metallic is able to go through while staying formed. In this specific posting, the sort capability of Ti6Al4V titanium alloy as well as Al6061 T6 tiny aluminum alloy sheets is really examined experimentally creating utilization of hydro actually being produced serious drawing [35].

Authors [36] simplified method was utilized to get experimental FLDs for these sheet substances. ANN modeling of the task based on experimental outcomes is in fact expelled to foresee FLDs. It is found out that a feed ahead once more back propagation ANN is able to count on the FLDs, thus, indicating the chance for ANN as a great system in simulating the progression. Based on comparisons there's a good agreement between neural and experimental society benefits. In this particular investigation work, a brand new ANN layout was created for forecasting the FLDs for Ti6Al4V titanium alloy as well as Al6061 T6 tiny aluminum alloy sheets. The experimental information was used for training the different neural networks versions. Based on experimental what about previous propagation neural society range of FLDs results the getting close to conclusions are in fact drawn:

(i) Average values for stress and anxiety hardening $n$ coupled with strain fee recognition are in fact $(0.145,3.02)$ also as $(0.17,0.57)$ for Al6016-T6 and Ti6Al4V sheets, respectively. As values of $\mathrm{N}$ along with $\mathrm{R}$ boost, the last strain advantage increases, and also as an outcome, the final elongation increases. This offers for your FLD to shift higher.

(ii) The results from Assessments and ANN style recommend the punch stroke, LDR, in addition to motor oil stress have pronounced influences on the little true strain and main true strain of Ti6Al4V titanium alloy as well as Al6061 T6 tiny light weight aluminum alloy sheets.

(iii) Based on probably the least instruction error, three different instruction algorithm methods are actually compared. It's been determined that the most effective strategy which performed least mistakes would be the Levenberg-Marquardt Algorithm (LMA).

(iv) Based on the portion typical teaching blunder along with proportion standard checking error, a number of architectures are in fact trained utilizing experimental info until a significant architecture is really driven. It was found that a way with a human being concealed amounts having four neurons to do as the very best society design \& design to foresee the FLDs of Ti6Al4V titanium alloy as well as Al6061 T6 tiny aluminum alloy sheets.

(v) The results of this specific research paper are in fact encouraging. The neural framework design worked well. It correctly predicted the FLD diagram which displays the practical usage of the neural phone system method to find out as well as solve issues which are complicated.

Published By:

Blue Eyes Intelligence Engineering \& Sciences Publication 
This particular certain method may also be regarded as a point to do virtual tests wherein the outcome of different variables might be evaluated, which occurs to be physically simply impossible.

This specific research newspaper [37] has a manmade ANN sort for predicting in addition to avoiding flooring catastrophe just like wrinkling of sheet metals.

Commercially wash little aluminum sheets of different levels are actually drawn into cylindrical cups by way of conical die. An ANN layout was created to map the actual physical characteristics and also instantaneous geometry functions of extreme drawing process. This specific style is actually a feed ahead returned propagation neural networking and it's taught also as experimented with pairs of inputs \& outputs information. A very great general functionality of neural network, around terminology of contract using the experimental info was achieved. This specific style is usually used for prediction and also analysis of originating of wrinkling. The primary conclusions that are drawn out of the current exploration are mentioned below:

(i) The multi level group with 2 concealed layers having 50 neurons, forty 8 neurons, as well as forty 6 neurons of the circumstance of light weight aluminum quality of sheets ISS 19000, ISS 19600, together with ISS 19660 respectively, coached with LMA had been found to run as the best community design developed in this specific analysis.

(ii) A great effectiveness is actually attained using the neural system model, with good correlation coefficient in between the item prediction along with the experimental values.

(iii) With the developed ANN model, it might be anticipated and additionally assessed the wrinkling behavior of fresh light weight aluminum quality sheets of ISS 19000, ISS 19600, too ISS 19660.

Within most the past few years, sheet metals are in fact made using perforations in patterns which are unique $\&$ kinds to have the ability to enhance the look of sheet and furthermore to protect extra fat of elements. As in normal metallic sheets, it's essential to create the perforated sheet metals moreover inside their secured strain elements to stay away from the growing issues as necking, fracture along with wrinkling. The FLD is really an excellent device to figure out the growing limit strains. The restricting strains of perforated sheet metals mostly rely on the geometry on the perforations along with acquiring variables. This results in big expansion of quantity of test to be finished with different geometry \& creating variables for selecting the increasing cap tension for perforated sheets. Aiming to take down the quantity of tests necessary, in this particular job, an ANN format was created for generating cap diagram of perforated $\mathrm{Al} 8011$ sheets seated on experimental outcomes along with correlated with the geometrical capabilities of perforated sheets. This particular like [38] is really a feed forward refunded propagation neural society making use of a pair of geometrical variables because the inputs of it's as well as the protected real strains as the outcome of its. This particular ANN design may be used for prediction of FLD of perforated sheet running a little bit of geometry. A report on obtaining cap diagram of perforated $\mathrm{Al} 8011$ sheets by experimental evaluation in addition to ANN is really carried through in this specific research newspaper. Create the foregoing discussion; the consequent conclusion may be made. The perforated Al 8011 sheets having two mm thickness show much better building cap strains and having a single mm thickness suggest lowered creating cap strains regardless of the geometrical capabilities of the perforated sheet along with the sheet owning two mm thickness, three mm size of perforation too as ten mm ligament wideness have larger like capability together with the sheet owning a single mm thickness, five mm size of perforation too as five mm ligament wideness have decreased approach capability in each and every one of the method of clear plastic made deformation, and there's a normal deviation of restrict anxiety ratio. The form capability of the perforated sheet is dependent on ligament ratio as well as area which are really open, so that as the ligament ratio advances and as of region that is readily available decreases, the form skill increases marginally. Determined by the correlation coefficient along with convergence, 306 extraordinary in previous propagation neural society architectures are in fact educated making use of the experimental information until a significant architecture is really driven and as a consequence of the various multi fitness level refunded propagation neural society architectures taught, the rear propagation neural society architectures with two concealed layers owning eighteen neurons in each and every (four - eighteen - eighteen - two) taught with LMA was discovered to be the ideal society design along with an excellent basic performance was accomplished with the neural system version, which demonstrates Positive Many Meanings great correlation between the probable values of the very best neural society like together with the experimental information for prediction of modest real strain and extensive real strain working with the whole information sets. For complete, the sort capability of perforated Al 8011 sheets originating from manmade neural modern society are really in great agreement with the experimental outcomes for picked thickness of this particular sheet dimensions of perforation as well as ligament width. Moreover the ANN is able to foresee the kind skill conduct of the blanks inside acceptable error limits.

This specific research newspaper [39] has a completely new method for fault analysis by way of a recently developed method, Support Vector Machine (SVM). First, the essential concept on the SVM is briefly assessed. Next, a rapid implementation algorithm is actually provided. Consequently the strategy is utilized for the fault examination of sheet metal stamping methods. Depending on the tests on 2 different instances, authors [39] are actually a blanking and additionally the additional individual is actually a progressive functioning, the completely new method is very beneficial. In both instances, the achievement rate of it is greater than $96.5 \%$. In comparison, the good results rate of the preferred ANN is actually 93.3\%. Furthermore, the novice driver method requires simply selection of instruction samples that is an attractive function for dealer floor apps. Based on the analysis provided previously, the following conclusions could be drawn:

Published By: 
(i) SVM is actually an incredibly fantastic device for fault diagnosis. it is a choice of appealing features: First, It is right and it's in a position to coping with unseen samples. Then, it is going to take just a bit of amount of instruction samples. Finally, it requires simple computation in decision making. These features permit it to be truly attractive for fault diagnosis.

(ii) The accomplishments of the SVM is mainly as a result of the reality that it will make use of a kernel attribute to map the indicators to a much better order choice region, in which additional characteristics of the signal may be recognized. Surprisingly, the choice of the kernel functionality is not vital supplied the capability of its enough.

(iii) Strain indicators of stamping methods are actually non-linear and non-stationary extremely, that's natural signs in engineering strategies. Depending on the 2 tests on the issue monitoring along with examination of sheet metallic stamping functions, the suggested SVM strategy achieved a success rate of in excess of $96.5 \%$. In comparability, the ANN with BPA attained simply ninety \% or perhaps a decreased quantity of this shows the SVM technique has a lot more capability of dependability as well as robustness. Authors [39] expected the presented state monitoring along with diagnosis strategy may be placed on to other manufacturing processes.

\section{PROPOSED MODEL}

The ANN based backpropagation learning model is implemented for mechanical engineering domain analysis. The architecture of the model defined through different layers i.e. 8-4-2. Input layers contain eight input nodes, hidden layers contain four nodes and output layers contains two nodes. The backpropagation algorithm used for training purpose.

\section{RESULTS}

The test results of ANN based proposed model indicating the utility and applicability of ANN in mechanical engineering domain. The total data of mechanical engineering domain is divvied in to three subgroups. The first subgroup contains training data. The second subgroup contains validation data and third subgroup contains testing data. Total 600 data collected from mechanical engineering domain companies and $60 \%$ data utilized for training. Rest $40 \%$ data divided into two equal parts i.e. 20\% data for validation purpose and $20 \%$ data for testing purpose. The training of ANN performed on training data. After training, the model is tune based on validation data. Finally the trained model is evaluated on test data. The test results of ANN shows $98.33 \%$ accuracy. The evaluation of absolutely no price literature in the region of functions of ANN to sheet metal jobs uncovers the method is really used by worldwide experts for particular programs as procedure managing, procedure preparation, quality managing, prediction of bending, gear page layout, function recognition, as well as also slicing gear option etc. However for virtually this entire app, seasoned people are important in an attempt to run the evolved program as well as additionally to purchase choices that are ideal from a lot of phases of sheet metallic provide the results. Within sheet metal industries, nevertheless it's thought the accomplishments of this particular defect totally no price type of sheet metal parts largely is determined by the adventure along with moreover, the ability of the merchandise customized. It appears that there's not sufficient reason treatments inside the evolved ANN uses to sheet metallic provide the results. In addition, literature is actually additionally not on the apps of ANN on the type of multi functioning media tools like for example for instance mixture kick the bucket, combination kick the pail, as well as next progressive kick the bucket that are in fact typical contained sheet metal industries.

\section{CONCLUSIONS}

Printed literature to the domain name of reasons of ANN to sheet metal do how about bodily engineering is really evaluated and also supplied as well as summarized to this evaluation newspaper. The great bulk of accessible exploration work is actually works with all of the prediction of sheet metallic bending approaches. Definitely no investigate worker's obligations are in fact stated associated with the use of ANN technique to develop of sheet metallic expires together with prediction of kick the container everyday life which will designers operating with sheet metal industries. Hence, there's stern should make use of ANN activity throughout these established locations. The evolved ANN activity for merchandise design along with kick the bucket design needs to regularly be unique welcoming, simple to work with as well as in addition have fairly great cost of put in position.

\section{REFERENCES}

1. Prieto, A., Prieto, B., Ortigosa, E. M., Ros, E., Pelayo, F., Ortega, J., \& Rojas, I. (2016). Neural networks: An overview of early research, current frameworks and new challenges. Neurocomputing, 214, 242-268.

2. Lee, D., Seo, H., \& Jung, M. W. (2012). Neural basis of reinforcement learning and decision making. Annual review of neuroscience, 35, 287-308.

3. Lesko, J. (2007). Industrial design: Materials and manufacturing guide. John Wiley \& Sons.

4. Mucha, J., Kaščák, L., \& Spišák, E. (2015). The experimental analysis of cold pressed joint technology for selected sheet metals used in an automotive industry. In Advanced Materials Research (Vol. 1077, pp. 33-38). Trans Tech Publications Ltd.

5. Myers, W. (1982). CAD/CAM: The need for a Broader Focus. Computer, (1), 105-117.

6. Feigenbaum, E. A. (1980). Knowledge Engineering: The Applied Side of Artificial Intelligence (No. STAN-CS-80-812). STANFORD UNIV CA DEPT OF COMPUTER SCIENCE.

7. Otto, K. N. (2003). Product design: techniques in reverse engineering and new product development. 清华大学出版社有限公司.

8. Kaur, K. (2009). Optical Multistage Interconnection Networks Using Neural Network Approach (Doctoral dissertation).

9. Fiesler, E., \& Beale, R. (Eds.). (2020). Handbook of neural computation. CRC Press.

10. Nordström, T., \& Svensson, B. (1992). Using and designing massively parallel computers for artificial neural networks. Journal of parallel and distributed computing, 14(3), 260-285.

11. Liu, G. P. (2012). Nonlinear identification and control: a neural network approach. Springer Science \& Business Media.

12. Downs, L. L. (1995). Manufacturing Inequality: Gender Division in the French and British Metalworking Industries, 1914-1939. Cornell University Press. 
13. Tolio, T., Bernard, A., Colledani, M., Kara, S., Seliger, G., Duflou, J., ... \& Takata, S. (2017). Design, management and control of demanufacturing and remanufacturing systems. CIRP Annals, 66(2), 585-609.

14. Mackerle, J. (2004). Finite element analyses and simulations of sheet metal forming processes. Engineering computations.

15. Gronostajski, Z., Hawryluk, M., Kaszuba, M., Marciniak, M. Niechajowicz, A., Polak, S., ... \& Durak, J. (2016). The expert system supporting the assessment of the durability of forging tools. The International Journal of Advanced Manufacturing Technology, 82(9-12), 1973-1991.

16. Sun, Y., Li, Y., Liu, Z., Daniel, W. J., Shi, L., Meehan, P. A., \& Ding, S. (2017). Experimental investigation and prediction of the maximum edge longitudinal membrane strain and springback of Chain-die-formed AHSS U-channels using response surface methodology. The International Journal of Advanced Manufacturing Technology, 90(5-8), 1963-1976.

17. Hussain, G., Gao, L., \& Zhang, Z. Y. (2008). Formability evaluation of a pure titanium sheet in the cold incremental forming process. The International Journal of Advanced Manufacturing Technology, 37(9-10), 920-926.

18. Thomas, W. J. (1999). Product, tool, and process design methodology for deep drawing and stamping of sheet metal parts (Doctoral dissertation, The Ohio State University).

19. Şenol, Ö., Esat, V., \& Darendeliler, H. (2014). Springback analysis in air bending process through experiment based artificial neural networks. Procedia Engineering, 81, 999-1004.

20. Wang, J., Wu, X., Thomson, P. F., \& Flitman, A. (2000). A neural networks approach to investigating the geometrical influence on wrinkling in sheet metal forming. Journal of Materials Processing Technology, 105(3), 215-220.

21. Wadi, I., \& Balendra, R. (1999). Using neural networks to model the blanking process. Journal of Materials Processing Technology, 91(1-3), 52-65.

22. Gisario, A., Barletta, M., Conti, C., \& Guarino, S. (2011). Springback control in sheet metal bending by laser-assisted bending: Experimental analysis, empirical and neural network modelling. Optics and Lasers in Engineering, 49(12), 1372-1383.

23. Khorasani, A. M. (2017). The selected laser melting production and subsequent post-processing of Ti-6Al-4V prosthetic acetabular (No. PhD). Deakin University.

24. McIntosh, G. (1998). The Johnson-Holmquist ceramic model as used in LS-DYNA2D (No. DREV-TM-9822). DEFENCE RESEARCH ESTABLISHMENT VALCARTIER (QUEBEC)

25. Zhou, X. (1999). Numerical prediction of springback in U-channel forming of aluminum tailor welded blanks (Doctoral dissertation, Carleton University).

26. Badrish, A., Morchhale, A., Kotkunde, N., \& Singh, S. K. (2020). Influence of material modeling on warm forming behavior of nickel based super alloy. International Journal of Material Forming, 1-21.

27. Benyelloul, K., \& Aourag, H. (2013). Elastic constants of austenitic stainless steel: Investigation by the first-principles calculations and the artificial neural network approach. Computational materials science, 67, 353-358.

28. Kumar, V. V., Yadav, S. R., Liou, F. W., \& Balakrishnan, S. N. (2013). A digital interface for the part designers and the fixture designers for a reconfigurable assembly system. Mathematical problems in engineering, 2013.

29. Lie, K. A. (2019). An introduction to reservoir simulation using MATLAB/GNU Octave: User guide for the MATLAB Reservoir Simulation Toolbox (MRST). Cambridge University Press.

30. Mansouri, L. Z., Chalal, H., \& Abed-Meraim, F. (2014). Ductility limit prediction using a GTN damage model coupled with localization bifurcation analysis. Mechanics of Materials, 76, 64-92.

31. Salandro, W. A., Bunget, C., \& Mears, L. (2011). Electroplastic modeling of bending stainless steel sheet metal using energy methods. Journal of manufacturing science and engineering, 133(4).

32. Liu, B. C. (2016). Joining dissimilar structural alloys by vaporizing foil actuator welding: process conditions, microstructure, corrosion, and strength (Doctoral dissertation, The Ohio State University).

33. Babu, K. V., Narayanan, R. G., \& Kumar, G. S. (2009). An expert system based on artificial neural network for predicting the tensile behavior of tailor welded blanks. Expert Systems with Applications, 36(7), 10683-10695.

34. Jeswiet, J., Geiger, M., Engel, U., Kleiner, M., Schikorra, M., Duflou, J., ... \& Bruschi, S. (2008). Metal forming progress since 2000. CIRP Journal of Manufacturing Science and Technology, 1(1), 2-17.
35. Odoh, D. O. O. (2012). Full Field Measurement of The Dynamic Response of AA6061-T6Aluminum Alloy under High Strain Rate Compression and Torsion Loads (Doctoral dissertation, Howard University).

36. Volk, W., Groche, P., Brosius, A., Ghiotti, A., Kinsey, B. L., Liewald, M., ... \& Yanagimoto, J. (2019). Models and modelling for process limits in metal forming. CIRP Annals, 68(2), 775-798.

37. Ruffini, R., \& Cao, J. (1998). Using neural network for springback minimization in a channel forming process. SAE transactions, 65-73.

38. TETI, R., SOLIMAN, A. A. A., SULIMAN, M., ENGEL, U., \& GRIZZUTI, N. Numerical Simulation of Metal Sheet Plastic Deformation Processes through Finite Element Method.

39. Ge, M., Xu, Y., \& Du, R. (2008). An intelligent online monitoring and diagnostic system for manufacturing automation. IEEE Transactions on Automation Science and Engineering, 5(1), 127-139.

\section{AUTHORS PROFILE}

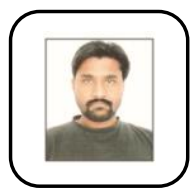

Prof. (Dr.) Mohit Gangwar is B.Tech., M.Tech. and Ph.D. (MNNIT, Allahabad) in Computer Science \& Engineering. Dr. Gangwar has more than 50 research publications. Dr. Gangwar is professional member of Association for Computing Machinery. He is working as Principal of Bhabha Engineering Research Institute, Bhopal, MP, India and Dean-Engineering of Bhabha University, Bhopal, MP, India.

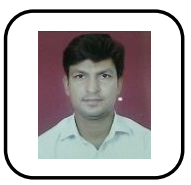

Mr. Brijesh Kumar Ojha is B.Tech. and M.Tech. He is working as Assistant Professor in Department of Mechanical Engineering, Faculty of Engineering, University of Lucknow, Lucknow, UP, India.

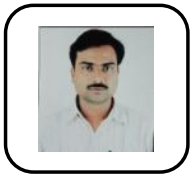

Mr. Rohit Srivastava is B.Tech. and M.Tech. He is working as Assistant Professor in Department of CSE, Faculty of Engineering, University of Lucknow, Lucknow, UP, India.

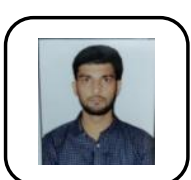

Mr. Himanshu Kumar Shukla is B.Tech. and M.Tech. He is working as Assistant Professor in Department of CSE, KMC Urdu-Arbi Farshi University, Lucknow, UP, India.

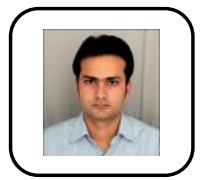

Mr. Aditya Pratap Singh is B.Tech. and M.Tech. He is working as Assistant Professor in Department of CSE, School of Management Science (AKTU Code 421), Lucknow, UP, India. 